Отримані аналітичні вирази для середньо-інтегральних по довжині коефічієнтів циліндричних гармонік магнітної індукції, створюваної надпровідною обмоткою всередині апертури дипольного або квадрупольного прямого електромагніту на основі геометричних параметрів обмотки. Показана пропорційність середньо-інтегральних вкладів в магнітну індукиію, створюваних неосновними гармоніками, середньо-інтегральному значенню основного поля

Ключові слова: пучок частинок, дипольний електромагніт, квадрупольна обмотка, циліндрична гармоніка, магнітна індукція

\section{Получены аналитические выражения для сред-} не-интегральных по длине коэффициентов цилиндрических гармоник магнитной индукции, создаваемой сверхпроводящей токовой обмоткой внутри апертуры дипольного или квадрупольного прямого электромагнита на основе геометрических параметров обмотки. Показана пропорциональность средне-интегральных вкладов в магнитную индукцию, создаваемых неосновными гармониками, средне-интегральному значению основного поля

Ключевые слова: пучок частии, дипольный электромагнит, квадрупольная обмотка, цилиндрическая гармоника, магнитная индукция
UDC 621.317.44

DOI: $10.15587 / 1729-4061.2018 .123607$

\title{
CYLINDRICAL \\ HARMONIC ANALYSIS OF THE MAGNETIC FIELD IN THE APERTURE OF THE SUPERCONDUCTING WINDING OF AN ELECTROMAGNET
}

A. Get man

$\mathrm{PhD}$, Senior Researcher

Department of Theoretical Electrical Engineering National Technical University

"Kharkiv Polytechnic Institute"

Kyrpychova str., 2, Kharkiv, Ukraine, 61002 E-mail: getmanav70@gmail.com

\section{Introduction}

In cyclic accelerators, electromagnets are traditionally used for transporting in an orbit and forming the transverse dimension of the particle beam. The provision of high-quality transportation and focusing is important from the point of view of the "lifetime" of the particle beam in the accelerator. The transverse magnetic field created by electromagnets in the aperture exerts a force on the particle beam. Depending on the spatial structure of the magnetic field of the electromagnet, the applied force action on the particles is equivalent to the effect of optical elements on the light beam. In this case, the deviations from the given law of distribution of the magnetic field in the aperture of the magnet are equivalent to the defects of the corresponding optical element (lens). When modelling the magnetic induction in the aperture of a dipole or quadrupole electromagnet, only two transverse projections are considered. In the aperture, the longitudinal (along the motion of the beam) component of the field created by the magnets exerts a substantially smaller force action on the charged particles. The transverse components of the magnetic induction inside the aperture are represented as a series of polar harmonics for each plane of the cross section [1]. This representation of the field makes it possible to specify the force action on the beam in the form of a convenient trajectory. The quantitative criterion for the quality of the electromagnetic field produced by the electromagnet is the total relative contribution from the harmonics that create an additional magnetic field.

\section{Literature review and problem statement}

The representation of magnetic induction in the form of a series [2] of polar harmonics for each plane of the cross section of an electromagnet has its peculiarity. The use of a number of polar harmonics is mathematically correct for infinitely long electromagnets. For real electromagnets of finite length, the use of a 2D magnetic field model based on polar harmonics is, generally speaking, an approximation, since, depending on the position of the viewing plane of the field relative to the centre of the electromagnet, the values of the harmonics coefficients change. In other words, the coefficients of the polar harmonics are not constant but a function of the longitudinal coordinate of the plane of the electromagnet section. The analytical 2D model of the magnetic field does not allow calculating the longitudinal variations of the coefficients of the polar harmonics. Therefore, $3 \mathrm{D}$ models based on the finite element method are used for their calculation. This procedure for calculating the longitudinal changes in the coefficients of the polar harmonics is not convenient in the case of solving the inverse problem, in particular, for optimizing the design with given field characteristics. In addition, when describing the force action of a magnetic field on a particle beam, the mean integral values of the polar harmonic coefficients are traditionally used [3]. The calculation of mean integral harmonics is based on integrating the results of Fourier analysis, which in turn uses data on the magnetic field obtained by the finite element method [4]. Such a complex chain of 3D analysis and calculation does 
not allow directly linking the longitudinal parameters of the design of the electromagnet with its magnetic characteristics, presented in the form convenient for calculating the force action on the particle beam [5]. The practical need for such interrelations between the parameters of the design of the electromagnet and its magnetic characteristics is evident both at the design stage and for customizing the samples already manufactured.

The magnetic field created by the electromagnet in the aperture can be represented in the form of two components - the field of the iron framework and the field of the current winding. When using electromagnets created according to the classical scheme with an iron framework but having a superconducting current winding, it is necessary to take into account the increased contribution (about onethird) to the total magnetic field from the latter.

Current winding of direct dipole and quadrupole magnets can be represented in the form of sets of rectilinear sections with current and end elements [6]. To calculate the magnetic field in the central cross section of the electromagnet, 2D models of magnetic induction are used [7], assuming rectilinear sections of the current winding to be infinitely long. Such an approximation does not fully allow taking into account the real geometry of the current winding and, therefore, can be used as an estimate and requires subsequent 3D calculation [8].

Therefore, it is of practical interest to create such models of the electromagnetic field of an electromagnet that allow matching some of the design parameters of an electromagnet with the mean integral length of the harmonics of magnetic induction harmonics [9].

For this purpose, it is expedient to perform a cylindrical harmonic analysis of the magnetic field produced by the superconducting current winding separately from the field of the iron framework. First of all, direct expressions for mean integral harmonics are of interest, making it possible to solve the inverse problem of searching for the optimal geometry of the superconducting winding. Considering the small (about ten) number of turns of the superconducting winding, it is rational to calculate the mean integral harmonics in the form of the sum of the winding contributions.

\section{The aim and objectives of the study}

The aim of the work is to construct a $3 \mathrm{D}$ model of the cylindrical harmonics of the magnetic field created by the current winding inside the aperture of a dipole (or quadrupole) direct electromagnet. The model will solve the problem of optimizing the design of an electromagnet by direct calculation of the mean integral lengths of the cylindrical harmonics of magnetic induction on the basis of the geometric parameters of the superconducting winding.

To achieve this goal, the following tasks must be solved in the work:

- to carry out a cylindrical harmonic analysis of the magnetic induction of the superconducting current winding of dipole and quadrupole electromagnets;

- to obtain direct expressions for the non-basic mean integral harmonics of the magnetic induction of the winding of the dipole and quadrupole electromagnets;

- to verify the obtained expressions by comparison with the results of a numerical calculation of the mean integral harmonics of the magnetic induction.

\section{Initial provisions and assumptions for a cylindrical harmonic analysis of magnetic induction}

Let us consider the magnetic field created by the superconducting winding of the dipole (Fig. 1, 2) or quadrupole (Fig. 3, 4) direct electromagnet.

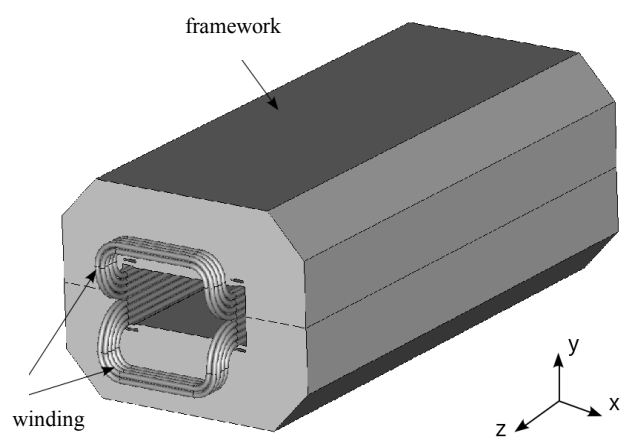

Fig. 1. A dipole electromagnet with superconducting winding

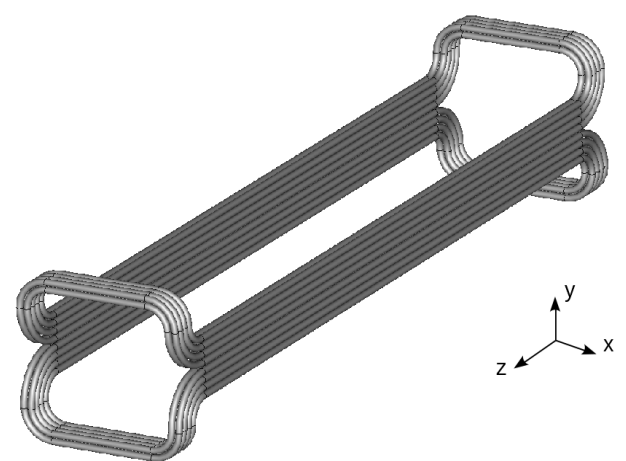

Fig. 2. Superconducting winding of the dipole electromagnet

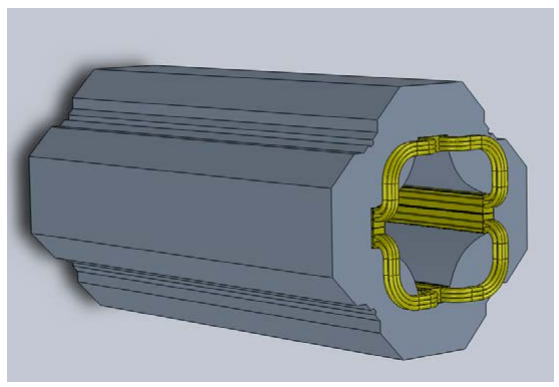

Fig. 3. A quadrupole electromagnet with superconducting winding

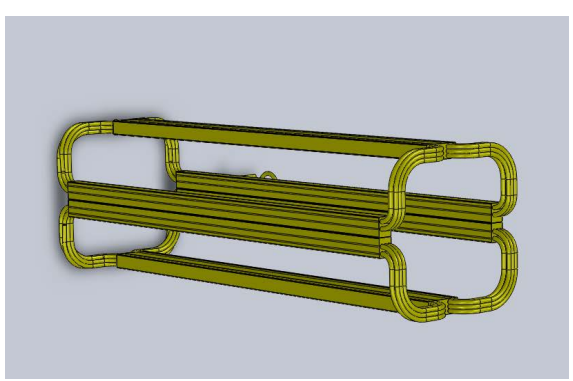

Fig. 4. Superconducting winding of the quadrupole electromagnet

For each cross-sectional plane, according to [2], the representation of the transverse projections of the magnetic induction $B_{\rho}$ and $B_{\varphi}$ is possible in the form of a series: 


$$
\begin{aligned}
& B_{\phi}(\rho, \phi, z)+i B_{\rho}(\rho, \phi, z)= \\
& =B_{0}(z) \sum_{n=1}^{\infty}\left[b_{n}(z)+i a_{n}(z)\right] \frac{(\rho)^{n-1}}{R_{r e f}^{n-1}} e^{i n \phi},
\end{aligned}
$$

where $\rho, \varphi, z$ are the cylindrical coordinates of the observation point of the magnetic field; $\mathrm{B}_{0}(\mathrm{z})$ is the main value of magnetic induction; $b_{n}(z)$ and $a_{n}(z)$ are the coefficients of the polar harmonics of the degree $n$.

The reference radius $R_{\text {ref }}$ is the radius of the circle, according to the magnetic induction data by which the coefficients of the polar harmonics were determined.

The mean integral values of the harmonics coefficients from (1) are introduced on the basis of [2]:

$$
\begin{aligned}
& b_{n}^{*}=\frac{\int_{-\infty}^{\infty} b_{n}(z) B_{0}(z) \mathrm{d} z}{\int_{-\infty}^{\infty} B_{0}(z) \mathrm{d} z}=\frac{\int_{-\infty}^{\infty} b_{n}(z) B_{0}(z) \mathrm{d} z}{B_{c} L_{e f f}}, \\
& a_{n}^{*}=\frac{\int_{-\infty}^{\infty} a_{n}(z) B_{0}(z) \mathrm{d} z}{\int_{-\infty}^{\infty} B_{0}(z) \mathrm{d} z}=\frac{\int_{-\infty}^{\infty} a_{n}(z) B_{0}(z) \mathrm{d} z}{B_{c} L_{e f f}},
\end{aligned}
$$

where $B_{c}$ is the main value of the magnetic induction in the central cross section of the electromagnet; $L_{\text {eff }}$ is the effective length of an electromagnet introduced as

$$
L_{e f f}=\frac{\int_{-\infty}^{\infty} B_{0}(z) \mathrm{d} z}{B_{c}} .
$$

To simplify the geometrical description of the winding, let us assume that the end elements lie in a plane perpendicular to the longitudinal axis of the magnet. In this case, the end elements of each turn are represented in the form of successively connected straight sections of an infinitely thin wire, for example, as shown for the quadrupole winding in Fig. 5.

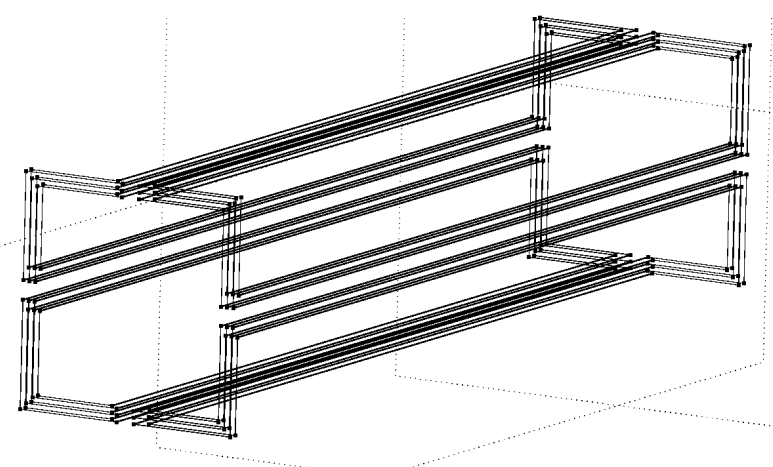

Fig. 5. Simplified representation of the elements of the current winding of a quadrupole electromagnet

In addition, let us first obtain a description of the magnetic field created by only one turn of the winding. This will eliminate the unprincipled index of the turn number in mathematical expressions. In the final representation of the result for the entire winding, let us introduce an additional summation index over all turns.
The simplified representation of the longitudinal elements of the turn in the form of rectilinear currents will be equally used for both the quadrupole and dipole cases, and it is first necessary to find for them a representation of the mean integral harmonics.

\section{The method of cylindrical harmonic analysis of the magnetic field created by longitudinal sections of the winding}

Let us obtain a representation for the mean integral harmonics produced by one longitudinal element of the coil of the current winding. Let the point of observation of the magnetic field always lie in the plane $z=0$ of the coordinate system whose centre lies on the longitudinal axis of the electromagnet.

Two projections of magnetic induction in a cylindrical coordinate system can be described on the basis of a single projection of the vector potential $A_{z}$ of the magnetic field [10].

$$
\begin{aligned}
& B_{\rho}(\rho, \phi, 0)=\frac{1}{\rho} \frac{\partial}{\partial \phi} A_{z}=\frac{\mu_{0} I}{4 \pi \rho} \int_{-L / 2}^{L / 2} \frac{\partial}{\partial \phi}\left(\frac{1}{\left|\vec{r}-\vec{r}_{0}\right|}\right) \mathrm{d} z_{0}, \\
& B_{\phi}(\rho, \phi, 0)=-\frac{\partial}{\partial \rho} A_{z}=\frac{\mu_{0} I}{4 \pi} \int_{-L / 2}^{L / 2} \frac{\partial}{\partial \rho}\left(\frac{1}{\left|\vec{r}-\vec{r}_{0}\right|}\right) \mathrm{d} z_{0} .
\end{aligned}
$$

On the other hand, the magnetic field in the aperture of the electromagnet can be represented on the basis of the spherical harmonics of the scalar potential [10]. Taking into account the relationship between the distance to the observation point and the distance to the elements with the current $r<r_{0}$, we use the internal solution for the scalar potential in a combined spherical coordinate system:

$$
\begin{aligned}
& B_{\rho}(\rho, \phi, 0)=-\frac{\partial}{\partial \rho}\left(\mu_{0} U\right)= \\
& =-\mu_{0} \frac{\partial}{\partial \rho}\left(\sum_{n=1}^{\infty} r^{n} \sum_{m=0}^{n} P_{n}^{m}(\cos \theta)\left\{\begin{array}{l}
a_{n}^{m} \cos m \phi \\
b_{n}^{m} \sin m \phi
\end{array}\right\}\right), \\
& B_{\phi}(\rho, \phi, 0)=-\frac{1}{\rho} \frac{\partial}{\partial \phi}\left(\mu_{0} U\right)= \\
& =-\frac{\mu_{0}}{\rho} \frac{\partial}{\partial \phi}\left(\sum_{n=1}^{\infty} r^{n} \sum_{m=0}^{n} P_{n}^{m}(\cos \theta)\left\{\begin{array}{c}
a_{n}^{m} \cos m \phi \\
b_{n}^{m} \sin m \phi
\end{array}\right\}\right),
\end{aligned}
$$

where $r, \theta, \varphi$ are the spherical coordinates of the observation point of the field.

It should be noted that of all spherical harmonics in (6) only the sectorial (for which $m=n$ ) satisfies the condition of zero longitudinal projection of the magnetic induction $\left(B_{z}=0\right)$. Sectorial harmonics have no dependence on the application, which follows from the representation of the associated Legendre functions on the basis of the Rodrigue formula in the form of a sum:

$$
\begin{aligned}
& r^{n} P_{n}^{m}(\cos \theta)=r^{m} r^{n-m}(\sin \theta)^{m} \sum_{s=0}^{n-m} c_{s}(\cos \theta)^{s}= \\
& =(r \sin \theta)^{m} \sum_{s=0}^{n-m} c_{s} r^{n-m}(\cos \theta)^{s}= \\
& =\rho^{m}\left(c_{n-m} z^{n-m}+\sum_{s=0}^{n-m-1} c_{s} r^{n-m}(\cos \theta)^{s}\right) .
\end{aligned}
$$


Therefore, in the case under consideration, the following representation holds:

$$
\begin{aligned}
& B_{\rho}(\rho, \phi, 0)=-\frac{\partial}{\partial \rho}\left(\mu_{0} U\right)= \\
& =-\mu_{0} \frac{\partial}{\partial \rho}\left(\sum_{n=1}^{\infty} \rho^{n}(2 n-1) ! !\left\{\begin{array}{l}
a_{n}^{n} \cos n \phi \\
b_{n}^{n} \sin n \phi
\end{array}\right\}\right), \\
& B_{\phi}(\rho, \phi, 0)=-\frac{1}{\rho} \frac{\partial}{\partial \phi}\left(\mu_{0} U\right)= \\
& =-\frac{\mu_{0}}{\rho} \frac{\partial}{\partial \phi}\left(\sum_{n=1}^{\infty} \rho^{n}(2 n-1) ! !\left\{\begin{array}{l}
a_{n}^{n} \cos n \phi \\
b_{n}^{n} \sin n \phi
\end{array}\right\}\right) .
\end{aligned}
$$

Using the representation of the inverse distance through spherical harmonics [10],

$$
\begin{aligned}
& \frac{1}{\left|\vec{r}-\vec{r}_{0}\right|}=\sum_{n=1}^{\infty} \frac{r^{n}}{r_{0}^{n+1}} \times \\
& \times\left(\begin{array}{l}
P_{n}\left(\cos \theta_{0}\right) P_{n}(\cos \theta)+ \\
+2 \sum_{m=1}^{n} \frac{(n-m) !}{(n+m) !} P_{n}^{m}\left(\cos \theta_{0}\right) P_{n}^{m}(\cos \theta) \cos m\left(\phi-\phi_{0}\right)
\end{array}\right),
\end{aligned}
$$

after substituting it in (5), for the coefficients of the spherical harmonics from (8), we can obtain the expression:

$$
\begin{aligned}
& \left\{\begin{array}{l}
a_{n}^{n} \\
b_{n}^{n}=-\frac{\mu_{0} I}{2 \pi} \frac{(2 n-1) ! !}{(2 n) !} \rho_{0}{ }^{n} \times
\end{array}\right. \\
& \times \int_{-L / 2}^{L / 2} \frac{\mathrm{d} z_{0}}{\left(\sqrt{\left(z_{0}+z_{c}\right)^{2}+\left(\rho_{0}\right)^{2}}\right)^{2 n+1}}\left\{\begin{array}{l}
\sin n \phi_{0}, \\
-\cos n \phi_{0},
\end{array}\right.
\end{aligned}
$$

where $z_{c}$ is the longitudinal coordinate of the centre of the rectilinear section $L$ with the current $I$.

After comparing the expressions for the coefficients from (1) and (8), we can write:

$$
\begin{aligned}
& \left\{\begin{array}{l}
a_{n}\left(z_{c}\right)=-n(2 n-1) ! ! \frac{R_{r e f}^{n-1}}{B_{0}\left(z_{c}\right)}\left\{\begin{array}{l}
a_{n}^{n} \\
b_{n}\left(z_{c}\right)
\end{array}=\right. \\
b_{n}^{n}
\end{array}\right. \\
& =\frac{\mu_{0} I}{2 \pi} \frac{n[(2 n-1) ! !]^{2}}{(2 n) !} \frac{R_{r e f}^{n-1}}{B_{0}\left(z_{c}\right)} \rho_{0}{ }^{n} \times \\
& \times \int_{-L / 2}^{L / 2} \frac{\mathrm{d} z_{0}}{\left(\sqrt{\left(z_{0}+z_{c}\right)^{2}+\left(\rho_{0}\right)^{2}}\right)^{2 n+1}}\left\{\begin{array}{l}
\sin n \phi_{0}, \\
-\cos n \phi_{0} .
\end{array}\right.
\end{aligned}
$$

By definition, the coefficients $b_{n}\left(z_{c}\right)$ and $a_{n}\left(z_{c}\right)$ have the meaning of the relative contribution to the magnetic induction of a non-fundamental harmonic of the degree $n$ in the cross-sectional plane at a distance $z_{c}$ from the centre of the electromagnet. For the fundamental harmonic, $n=$ main of the normal electromagnet, the coefficient $b_{\text {main }}(z)$ is one (for $\left.a_{\text {main }}(z)=0\right)$, and for skew-electromagnet $a_{\text {main }}(z)$ is equal to one (for $b_{\text {main }}(z)=0$ ).

\section{Cylindrical harmonics of the magnetic field of the dipole winding}

For the case of the main field of a normal dipole electromagnet, we immediately obtain from (11) the following:

$$
B_{0}\left(z_{c}\right)=-\frac{\mu_{0} I \rho_{0} \cos \phi_{0}}{4 \pi} \int_{-L / 2}^{L / 2} \frac{\mathrm{d} z_{0}}{\left(\sqrt{\left(z_{0}+z_{c}\right)^{2}+\rho_{0}^{2}}\right)^{3}} .
$$

Whence for the central cross-section with $z_{c}=0$ it is possible to obtain the value of the ground field created by one longitudinal rectilinear section of the winding of the dipole winding:

$$
B_{0}(0)=-\frac{\mu_{0} I \cos \phi_{0}}{2 \pi \rho_{0}} \frac{L}{\sqrt{L^{2}+\left(2 \rho_{0}\right)^{2}}} .
$$

Accordingly, the effective length can be represented on the basis of the integral in (4) in the form:

$$
L_{e f f}=\frac{\int_{\infty}^{-\infty} B_{0}\left(z_{c}\right) d z_{c}}{B_{0}(0)}=\sqrt{(L)^{2}+\left(2 \rho_{0}\right)^{2}} .
$$

The contribution to the mean integral harmonic of the degree $n$ on the basis of (2) after integration can be represented as:

$$
b_{n}^{*}=\left(\frac{R_{r e f}}{\rho_{0}}\right)^{n-1} \frac{\cos n \phi_{0}}{\cos \phi_{0}} .
$$

Expressions (11)-(15) obtained refer only to the direct wire of the coil of the dipole winding with the coordinates $\left(\rho_{0} ; \varphi_{0} ; z_{c}-L / 2, z_{c}+L / 2\right)$. We take into account that in the dipole winding there is always a reverse wire (with a current in the opposite direction), but having the coordinates $\left(\rho_{0} ; \varphi_{0}+\pi ; z_{c}-L / 2, z_{c}+L / 2\right)$, by virtue of the symmetry, we consider such a pair to be one coil. If we sum the numerator and the denominator from (2) over all the turns, substituting the coordinates of the straight wires, we obtain the relative contribution of the mean integral harmonic of the degree $n$ (not the ground field) from the longitudinal elements of the dipole current winding to the magnetic induction. Finally, for a dipole winding consisting of $w$ turns, we obtain an expression for the relative mean integral coefficients of the harmonics of the magnetic induction:

$$
b_{n}^{*}=\frac{\sum_{w} \frac{L_{w}}{\rho_{0 w}}\left(\frac{R_{r e f}}{\rho_{0 w}}\right)^{n-1} \cos n \phi_{0 w}}{\sum_{w} \frac{L_{w}}{\rho_{0 w}} \cos \phi_{0 w}} .
$$

With an ideal arrangement of the windings of the dipole winding, the total magnetic field from the longitudinal sections contains only harmonics of odd powers $n=2 k+1$, where $k=0,1,2 \ldots$

\section{Cylindrical harmonics of the magnetic field of the quadrupole winding}

By analogy, we obtain expressions for the case of the winding of a normal quadrupole electromagnet. Taking into account that for the quadrupole case the main field is described by the harmonic of the degree of main $=2$ from (11), then for one longitudinal rectilinear section with the coordinates $\left(\rho_{0} ; \varphi_{0} ; z_{c}-L / 2, z_{c}+L / 2\right)$ of the current winding, 


$$
B_{0}\left(z_{c}\right)=-\frac{3 \mu_{0} I R_{r e f}}{8 \pi} \cos 2 \phi_{0} \int_{-\frac{L}{2}}^{\frac{L}{2}} \frac{\rho_{0}^{2} \mathrm{~d} z_{0}}{\left(\sqrt{\left(z_{0}+z_{c}\right)^{2}+\rho_{0}^{2}}\right)^{5}} .
$$

From (17), by substituting the distance $z_{c}=0$ from the plane of consideration of the field for the central cross section of the electromagnet, we immediately obtain an expression for the principal value of the magnetic induction at the reference radius:

$$
B_{0}(0)=-\frac{\mu_{0} I L R_{r e f} \cos 2 \phi_{0}}{2 \pi \rho_{0}^{2}} \frac{L^{2}+6\left(\rho_{0}\right)^{2}}{\left(\sqrt{L^{2}+\left(2 \rho_{0}\right)^{2}}\right)^{3}} .
$$

By analogy with (14), the effective length of the current section in the quadrupole harmonic is determined from the expression:

$$
L_{e f f}=\frac{\left(\sqrt{L^{2}+\left(2 \rho_{0}\right)^{2}}\right)^{3}}{L^{2}+6\left(\rho_{0}\right)^{2}} .
$$

The relative contribution to the mean integral harmonic of the degree $n$ on the basis of (2) after integration can be represented as

$$
b_{n}^{*}=\left(\frac{R_{r e f}}{\rho_{0}}\right)^{n-2} \frac{\cos n \phi_{0}}{\cos 2 \phi_{0}} .
$$

A feature of the symmetry of the quadrupole winding is the presence of two straight and two return wires located through the angle $\pi / 2$ in such a way that by choosing as the initial straight wire with the coordinates $\left(\rho_{0} ; \varphi_{0} ; z_{c}-L / 2\right.$, $z_{c}+L / 2$ ), expression (20) is valid for the entire four straight longitudinal sections of the winding (equivalent to one turn). Summing over all the w turns of the winding and substituting in (2), we obtain a relative mean integral contribution to the magnetic induction of the ground field from the harmonic of the degree $n$ created by the entire quadrupole winding:

$$
b_{n}^{*}=\frac{\sum_{w} \frac{L_{w}}{\rho_{0 w}}\left(\frac{R_{r e f}}{\rho_{0 w}}\right)^{n-1} \cos n \phi_{0 w}}{\sum_{w} \frac{L_{w} R_{r e f}}{\left(\rho_{0 w}\right)^{2}} \cos 2 \phi_{0 w}} .
$$

It should be noted that in the case of an ideally precise arrangement of the longitudinal sections of the quadrupole winding, the spectrum of the created cylindrical harmonics will contain only the ones having the degree $n=2+4 k$, where $k=0,1,2 \ldots$, and all the skew harmonics are absent.

\section{Cylindrical harmonics of the magnetic field created by the end elements of the winding}

Let us represent the end elements of the current winding in a simplified form, as shown in Fig. 5. For each turn, the end elements are rectilinear segments of an infinitely thin wire oriented perpendicular to each other. Then the vector potential written for one such rectilinear portion of the end of the winding will have only one Cartesian projection $A_{x}$ if the region is parallel to the axis of abscissas, or $A_{y}$ if the section is parallel to the y-axis. Accordingly, in magnetic induction, only two Cartesian projections will be different from zero. Let us consider, for definiteness, the winding section parallel to the ordinate axis $\left(y_{1}=0, y_{2}=0.135\right)$. Then the transverse axis of symmetry of the magnet of the magnetic induction component $B_{x}$ will be an odd function of the longitudinal coordinate $z$ (Fig. 6).

Correspondingly, the integral of this projection, taken in the symmetric limits $\pm \infty$, will be equal to zero:

$$
\begin{aligned}
& \int_{-\infty}^{+\infty} B_{x} d z=\int_{-\infty}^{+\infty}\left(-\frac{\partial}{\partial z} A_{y}\right) \mathrm{d} z= \\
& =-\frac{\mu_{0} I}{4 \pi} \int_{-\infty}^{+\infty} \frac{\partial}{\partial z} \int_{y^{\prime}}^{y^{\prime \prime}} \frac{\mathrm{d} y_{0}}{\sqrt{\left(x-x_{0}\right)^{2}+\left(y-y_{0}\right)^{2}+\left(z-z_{0}\right)^{2}}} \mathrm{~d} z .
\end{aligned}
$$

Accordingly, the contributions to the mean integral harmonics from all the end elements of the current winding will be equal to zero.

\section{Verification and discussion of the results obtained by} cylindrical harmonic analysis and numerical calculation

To verify the obtained analytical expressions for the mean integral coefficients of harmonics, their numerical calculation was performed based on (2). Typical values for the parameters of the superconducting winding of a dipole electromagnet were used. The current in the winding was $10 \mathrm{kA}$, the length of the linear part of the winding was $L=1 \mathrm{~m}$, the polar coil coordinates were $\left(\rho_{0}=0.14 \mathrm{~m}, \varphi_{0}=5^{\circ}\right)$, and $R_{\text {ref }}=0.07 \mathrm{~m}$. The values of the parameters were substituted in the expression:

$$
b_{n}^{*}=\frac{\int_{-\infty}^{\infty}\left(\int_{0}^{2 \pi} B_{\phi}\left(R_{r e f}, \phi, z\right) \cos (n \phi) \mathrm{d} \phi\right) \mathrm{d} z}{\int_{-\infty}^{\infty}\left(\int_{0}^{2 \pi} B_{\phi}\left(R_{r e f}, \phi, z\right) \cos (\phi) \mathrm{d} \phi\right) \mathrm{d} z} .
$$

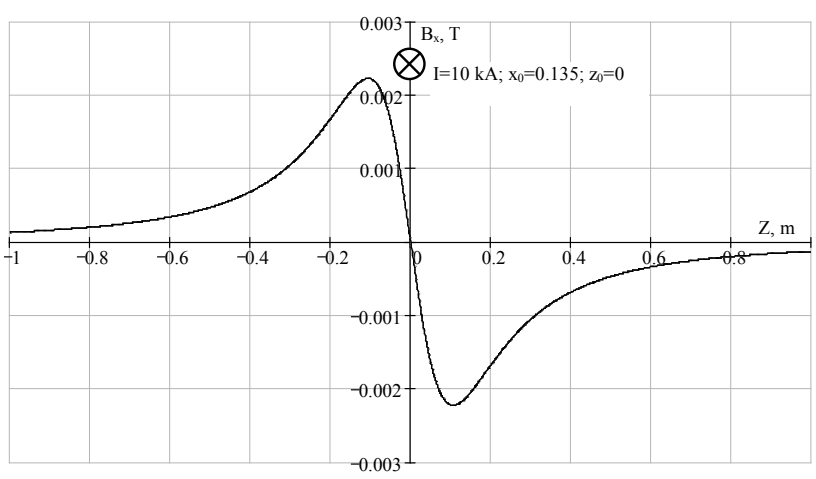

Fig. 6. Dependence of the transverse projection of the magnetic induction created by one rectilinear current element of the end section of the winding

For the calculation from (23), the expression for the angular projection of the magnetic induction $\mathrm{B}_{\varphi}$ from (5) was used as follows: 


$$
\begin{aligned}
& B_{\phi}\left(R_{r e f}, \phi, z\right)=\frac{\mu_{0} I}{4 \pi} \int_{-L / 2}^{L / 2} \frac{\partial}{\partial \rho}\left(\frac{1}{\left|\vec{r}-\vec{r}_{0}\right|}\right) \mathrm{d} z_{0}= \\
& =\frac{\mu_{0} I}{4 \pi} \int_{-L / 2}^{L / 2} \frac{\rho_{0} \cos \left(\phi-\phi_{0}\right)-R_{r e f}}{\left(\rho_{0}{ }^{2}+\left(R_{r e f}\right)^{2}-2 \rho_{0} R_{r e f} \cos \left(\phi-\phi_{0}\right)+\left(z-z_{0}\right)^{2}\right)^{3 / 2}} \mathrm{~d} z_{0} .
\end{aligned}
$$

The difference between the results of the analytical and numerical calculations given in Table 1 is close to the error in the computations.

Table 1

The mean integral coefficients of harmonics obtained by means of analytical and numerical calculation

\begin{tabular}{|c|c|c|c|}
\hline $\begin{array}{c}\text { Harmonic } \\
\text { coefficient }\end{array}$ & $\begin{array}{c}\text { Analytical } \\
\text { calculation }\end{array}$ & $\begin{array}{c}\text { Numerical } \\
\text { calculation }\end{array}$ & Relative error \\
\hline $\mathrm{b}_{3}^{*}$ & 0.242403877 & 0.24240412 & $0.0001 \%$ \\
\hline $\mathrm{b}_{5}^{*}$ & 0.05686067 & 0.05686061 & $0.0001 \%$ \\
\hline $\mathrm{b}_{7}^{*}$ & 0.01284816 & 0.01284814 & $0.0001 \%$ \\
\hline
\end{tabular}

The expressions obtained for the mean integral values of the coefficients of the non-fundamental harmonics of the magnetic induction produced by the dipole (16) and quadrupole (21) windings make it possible to take into account the actual length of the windings of the superconducting winding. The obtained results are in good agreement with the analogous formulae of the known two-dimensional model [11].

It is obvious that the condition for arranging the end elements of the winding in a plane perpendicular to the longitudinal axis of the electromagnet is sufficient to exclude their contributions to the mean integral harmonics. Also, the effect of the magnetization of the end elements of the iron framework is obvious, which should lead to the appearance of "uncharacteristic" harmonics of magnetic induction. However, it is possible to calculate such influence only by numerical methods.
The practical value of the obtained analytical representations is that they help calculate the necessary correction of the geometric parameters of the current winding. This need arises when optimizing the magnetic field inside the aperture of dipole and quadrupole electromagnets based on the given mean integral coefficients.

\section{Conclusions}

1. The study has shown the possibility of representing, in the form of a series of polar harmonics, a plane magnetic field produced by longitudinal sections of a dipole (or quadrupole) winding with a current of $10 \mathrm{kA}$. The obtained representation of the magnetic field is based on the spherical sectorial harmonics of the internal solution for the scalar potential, taken with coefficients depending on the longitudinal coordinate.

2. Analytical expressions have been obtained for the mean integral coefficients of the cylindrical harmonics of the magnetic induction produced by the superconducting current winding of the dipole and quadrupole electromagnets. The proportionality of the main field of the mean integral contributions to the magnetic induction produced by the non-fundamental harmonics has been shown.

3 . The article reflects the complexity of taking into account contributions to the mean integral values of the harmonics coefficients from the end elements of the winding, since these contributions arise only in the presence of an iron framework, as a result of its magnetization.

4. The obtained analytical representations of the mean integral coefficients of the harmonics of magnetic induction make it possible to optimize the magnetic field having a value of $1.5 \mathrm{~T}$ within the aperture of the dipole and quadrupole electromagnets. The necessary correction of the geometrical parameters of the current winding can be calculated with an error of not worse than $10^{-5}$ with respect to the given values of the mean integral harmonics of the magnetic induction.

Referenses

1. Russenschuck S., Tortschanoff T. Mathematical optimization of superconducting accelerator magnets // IEEE Transactions on Magnetics. 1994. Vol. 30, Issue 5. P. 3419-3422. doi: 10.1109/20.312673

2. Russenschuck S. Differential Geometry Applied to Coil-End Design // Field computation for accelerator magnets. Wiley-VCH Verlag GmbH \& Co. KGaA, 2010. P. 609-636. doi: 10.1002/9783527635467.ch19

3. Design Challenges for a Wide-Aperture Insertion Quadrupole Magnet / Russenschuck S., Auchmann B., Perez J. C., Ramos D., Fessia P., Karppinen M. et. al. // IEEE Transactions on Applied Superconductivity. 2011. Vol. 21, Issue 3. P. 1674-1678. doi: 10.1109/tasc.2011.2105453

4. Fischer E., Khodzhibagiyan H. G., Kovalenko A. D. Full Size Model Magnets for the FAIR SIS100 Synchrotron // IEEE Transactions on Applied Superconductivity. 2008. Vol. 18, Issue 2. P. 260-263. doi: 10.1109/tasc.2008.922261

5. Full size prototype magnets for heavy ion superconducting synchrotron SIS100 at GSI: status of manufacturing and test at JINR / Kovalenko A., Agapov N., Alfeev A. et. al. // EPAC'08. Genoa, 2008. P. 2443-2445.

6. Numerical Analysis of the Operation Parameters of Fast Cycling Superconducting Magnets / Fischer E., Schnizer P., Kurnyshov R., Schnizer B., Shcherbakov P. // IEEE Transactions on Applied Superconductivity. 2009. Vol. 19, Issue 3. P. 1266-1269. doi: 10.1109/ tasc.2009.2018746

7. Magnetic Field Measurement for Synchrotron Dipole Magnets of Heavy-Ion Therapy Facility in Lanzhou / Yang W., Zhang X., Han S., Yang J., Pei C., Yang L. et. al. // IEEE Transactions on Applied Superconductivity. 2014. Vol. 24, Issue 3. P. 1-4. doi: 10.1109/ tasc.2013.2289953

8. Magnetic Field Design of the Dipole for Super-FRS at FAIR / Yao Q. G., Ma L. Z., Zhang X. Q., He Y., Wu W., Moritz G. et. al. // IEEE Transactions on Applied Superconductivity. 2010. Vol. 20, Issue 3. P. 172-175. doi: 10.1109/tasc.2009.2038890

9. Magnetic Field Analysis for Superferric Accelerator Magnets Using Elliptic Multipoles and Its Advantages / Schnizer P., Schnizer B., Akishin P., Fischer E. // IEEE Transactions on Applied Superconductivity. 2008. Vol. 18, Issue 2. P. 1605-1608. doi: 10.1109/ tasc.2008.920636

10. Vanderlinde J. Classical electromagnetic theory. Springer, 2005. 420 p. doi: 10.1007/1-4020-2700-1

11. Wolff S. Superconducting accelerator magnet design // AIP Conference Proceedings. 1992. Vol. 249. doi: 10.1063/1.41989 